\title{
Multiple View Reconstruction of People
}

\author{
Adrian Hilton \& Jonathan Starck \\ Centre for Vision, Speech and Signal Processing \\ University of Surrey, Guildford, GU2 7XH, UK \\ a.hilton@surrey.ac.uk \\ http://www.ee.surrey.ac.uk/CVSSP/VMRG
}

\begin{abstract}
This paper presents a unified framework for model-based and model-free reconstruction of people from multiple camera views in a studio environment. Shape and appearance of the reconstructed model are optimised simultaneously based on multiple view silhouette, stereo and feature correspondence. A priori knowledge of surface structure is introduced as regularisation constraints. Model-based reconstruction assumes a known generic humanoid model a priori, which is fitted to the multi-view observations to produce a structured representation for animation. Model-free reconstruction makes no priori assumptions on scene geometry allowing the reconstruction of complex dynamic scenes. Results are presented for reconstruction of sequences of people from multiple views. The model-based approach produces a consistent structured representation, which is robust in the presence of visual ambiguities. This overcomes limitations of existing visual-hull and stereo techniques. Model-free reconstruction allows high-quality novel viewsynthesis with accurate reproduction of the detailed dynamics for hair and loose clothing. Multiple view optimisation achieves a visual quality comparable to the captured video without visual artefacts due to misalignment of images.
\end{abstract}

\section{Introduction}

People are central to most entertainment and communication content. Conventional 2D video production technologies use chroma-key to achieve video-realistic compositing of people within $2 \mathrm{~d}$ background scenes. However, direct compositing of the captured 2D video restricts rendering to a fixed viewpoint and limits interaction between the $2 \mathrm{D}$ video of a person and the surrounding scene. The challenge for computer vision is to construct representations of real people which allow video-realistic compositing within $2 \mathrm{D}$ and $3 \mathrm{D}$ scenes to produce novel video sequences. Representations should support manipulation of the viewpoint, illumination and movement of a person together with rendering of image sequences with the visual quality of conventional $2 \mathrm{D}$ video.

Over the past decade there has been extensive research in computer vision and graphics focused on modelling the appearance and movement of real people. Approaches to modelling real people include 3D scanning [6, 1], reconstruction from static images [7, 5], multiple view images [15, 2] and reconstruction from multiple view video [9, 11, 16]. Kanade et al. [9] and Moezzi et al. [11] introduced the reconstruction of sequences of people from multiple view video. Multiple view stereo reconstruction was used [9, 12] to reconstruct $3 D$ video sequences of people captured using a 51 camera system. This allowed a Virtualized Reality visualisation of the event in 3D with arbitrary viewpoint. Silhouette based reconstruction of the visualhull [11, 20] from multiple view video with $8-10$ cameras has also been applied to reconstruction of moving people. Video-rate reconstruction using silhouettes has been achieved using an image-based representation of the visual hull [10]. Silhouette and stereo based approaches make no prior assumptions of the observed scene topology or geometry enabling reconstruction of complex dynamic scenes such as moving people with loose clothing. However, the reconstruction for both approaches is not accurate resulting in visual artefacts in the rendered images of a scene. The visual-hull requires a large number of views to accurately reconstruct a scene and does not reconstruct concavities. Stereo reconstruction fails in regions of uniform appearance and close to silhouette edges. Therefore the results of these approaches although realistic contain visual artefacts which limit their application in broadcast or film production.

Model-based reconstruction approaches have recently been introduced [5, 2, 15, 13] which assume a prior model of the scene structure. A generic humanoid model representing the shape and articulation structure of a person is introduced to reconstruct the scene from multiple views. Multiple view silhouette [5] and stereo [13] observations are used to deform the generic model to the shape and pose of a 
specific person. This approach provides a consistent model structure for image sequences which can subsequently be used for animation. However, model-based approaches are limited to reconstruction of scenes which are close to the model structure. Current model based approaches are therefore restricted to relatively tight clothing and short hair unless these are specifically introduced into the model.

In this paper we present a unified approach to multiple view optimisation using either a model-free [9, 11, 10, 20] or model-based [5, 2, 15, 13] approach. Multiple view optimisation allows the integration of stereo and silhouette information in the reconstruction to overcome the limitations of each approach. Model-free reconstruction, presented in section 3, demonstrates that multiple view optimisation allows reconstruction of complex dynamic scenes with a visual quality approaching the captured images. Model-based reconstruction, presented in section 4 , using multiple view optimisation produces structured models which integrate the information from all available views. The resulting texture mapped models avoid visual artefacts but are limited to reconstruction of scenes with similar structure to the generic model.

\section{Multiple View Reconstruction Framework}

This section presents a framework for multiple view optimisation of shape and appearance. Application of this approach to model-based reconstruction was previously presented in [15]. The framework combines observations from silhouette, stereo and feature correspondence to reconstruct a surface which minimises the reprojection error. This approach combines gradient descent to minimise an objective function with stereo correspondence search to avoid local minima. Previous multiple view optimisation algorithms [4, 3, 17] using gradient descent converge to the nearest local minima which depends on the initial conditions.

Given an initial estimate of the surface shape we optimise the surface to satisfy an objective function with two data terms. The cost of fitting the multiple view silhouette data $E_{V}$ and fitting the stereo data $E_{S}$. A third data term to satisfy explicit point feature constraints has also been used in previous work[15]. To achieve reliable reconstruction for uniform image regions where stereo fails the data fitting energy $E_{D}=E_{V}+E_{S}$ is weighted according to the local variation in surface apperance. A regularisation term $E_{R}$ is also introduced to constrain the reconstructed surface shape according to an a priori model or assumption of surface smoothness. Energy minimisation of the objective function is performed using gradient descent:

$$
\begin{gathered}
E=E_{V}+E_{S}+\alpha E_{R} \\
\frac{d \underline{x}_{v}}{d t}=-\frac{d E}{d \underline{x}_{v}}=-\left(\frac{d E_{V}}{d \underline{x}_{v}}+\frac{d E_{S}}{d \underline{x}_{v}}+\alpha \frac{d E_{R}}{d \underline{x}_{v}}\right)
\end{gathered}
$$

The data fitting terms are defined as squared errors between the surface and the corresponding observation (silhouette, stereo or feature). This gives a least-squares minimisation which is solved using standard gradient descent procedures in a multi-resolution coarse-to-fine approach [15]. The principal contribution of our approach is the introduction of a search for good correspondence in the minimisation. Previous algorithms [4, 3] directly reproject the surface into the observed images to evaluate the error resulting in convergence to the nearest local minima to the initial estimate. The introduction of search to find the best correspondence within the expected error for the surface reconstruction avoids local minima due to noise and incorrect local matches. This search is performed in a coarse to fine manor, allowing refinement of the reconstruction to give sub-pixel reprojection error from stereo correspondence.

In this work the surface is represented as a mesh with a discrete set of vertices $\underline{x}_{v}$. Optimisation according to Equation 1 is performed at each mesh vertex. Multi-resolution mesh subdivision is used to refine the surface representation during optimisation according to the expected estimation error. Further details of the surface reconstruction algorithm implementation and evaluation can be found in [15, 14].

\subsection{Stereo Matching}

Standard stereo matching algorithms use epi-polar geometry to perform a constrained search for correspondences between adjacent pairs of images. In this work we apply the standard stereo search for surface optimisation. Estimated surface points are re-projected to adjacent images to search for the best correspondence.

Initially we determine the set of views for which the estimated surface point $\underline{x}_{v}$ is unoccluded. For each estimated surface point we then determine the key-view image with best viewpoint as the camera with the closest viewing direction to the surface normal. Stereo matching is then performed between the project point in the key-view and adjacent camera views. The ray from the key-view camera centre to the estimated surface point is projected to adjacent camera views to obtain the epipolar constraint. Stereo search is then performed along the epipolar line with the range determined by the surface estimation error. This error is refined in a coarse-to-fine optimisation as part of the surface optimisation of Equation 11. The search range perpendicular to the epi-polar line is defined by the maximum reprojection error of the camera calibration. Normalised cross-correlation with a fixed window size is used to perform the matching [15].

For each surface point we obtain a reconstructed 3D position from the disparity between the key-view and each adjacent camera $c$ from which the surface point is visible. We define our stereo matching energy term at each surface point 
$\underline{x}_{v}$ and the squared error between the estimated surface position and each of the reconstructed 3D positions $\underline{z}_{v, c}$ as follows:

$$
\begin{aligned}
& E_{S}=\sum_{v} \frac{1}{n_{v}^{c}} \sum_{c=0}^{n_{v}^{c}-1}\left\|\underline{z}_{v, c}-\underline{x}_{v}\right\|^{2} \\
& \frac{d E_{S}}{d \underline{x}_{v}}=-\frac{1}{n_{v}^{c}} \sum_{c=0}^{n_{v}^{c}-1}\left(\underline{z}_{v, c}-\underline{x}_{v}\right)
\end{aligned}
$$

Stereo matching gives sub-pixel correspondence between the key-view and each adjacent views for image regions with non-uniform appearance. The stereo correspondence constraint therefore allows the reconstruction of surfaces which align the observed appearance across multiple images upto the camera calibration error (typically $<1$ pixel).

\subsection{Sihouette Constraints}

Stereo requires variation in local surface appearance to obtain correspondence between multiple views. In uniform surface regions stereo does not provide reliable estimates of surface shape. The visual-hull gives a robust upper bound on the volume occupied by the object which can be used to constrain the shape in regions of uniform appearance. In this work the visual-hull is explicitly reconstructed using a discrete voxel representation (typically with $1 \mathrm{~cm}$ resolution corresponding to the pixel size). Surface points $\underline{x}_{v}$ are then constrained to fit the closest-point on the discrete visual-hull $\underline{y}_{v}:$

$$
\begin{aligned}
& E_{V}=\sum_{v}\left\|\underline{y}_{v}-\underline{x}_{v}\right\|^{2} \\
& \frac{d E_{V}}{d \underline{x}_{v}}=-\left(\underline{y}_{v}-\underline{x}_{v}\right)
\end{aligned}
$$

Explicit feature constraints can similarly be included in the formulation by introducing target 3D feature points which must be satisfied by the reconstructed surface [15]. This allows user-specified constraints to be included for key features such as the eyes and mouth which may contain insufficient detail to be matched by the stereo algorithm at standard image resolution.

\subsection{Combining Stereo and Silhouette data}

Robust reconstruction requires the combination of stereo matching constraints in surface regions with a local variation in appearance with silhouette constraints in uniform regions. The data energy term for model optimisation is formulated to incorporate both the stereo and silhouette data terms with a weight for each surface point, $0 \leq \mu_{i} \leq$

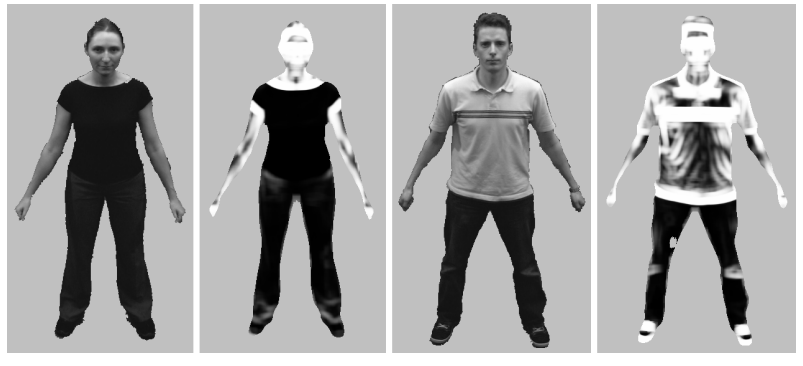
(a) Key image
(b) $\mu_{i}$ image
(c) Key image
(d) $\mu_{i}$ image

Figure 1. The weighting score for stereo matching shown across two key images with a $13 \times 13$ image window.

1 , defining the trade-off between the two complementary shape cues.

$E_{D}=\sum_{i=1}^{N_{v}} \mu_{i} \frac{1}{n_{v}^{c}} \sum_{c=0}^{n_{v}^{c}-1}\left\|\underline{z}_{v, c}-\underline{x}_{v}\right\|^{2}+\left(1-\mu_{i}\right)\left\|\underline{y}_{v}-\underline{x}_{v}\right\|^{2}$

The relative weighting $\mu_{i}$ represents a confidence measure in the stereo correspondence recovered at a vertex with respect to a key camera view. The normalised correlation score at a stereo match could be considered as a confidence score, however a high correlation can be obtained in matching regions of low image texture where the correspondence is ambiguous. The confidence measure is defined according to the ability to recover good correspondence where there is a high degree of intensity variation in the key camera view. A simple measure is defined in which the standard deviation $\sigma_{i}$ of the pixel intensities at the projected vertex location $\underline{u}_{i}$ in the key camera view is used.

$$
\sigma_{i}=\sqrt{\sum_{\underline{w}}\left(I\left(\underline{u_{i}}+\underline{w}\right)-\bar{I}\left(\underline{u_{i}}\right)\right)^{2}}
$$

This score is converted to the weight $\mu_{i}$ with a transformation based on a predefined point $\sigma_{0.5}$ where equal weighting is given to both the silhouette and stereo data $\mu_{i}=0.5$.

$$
\mu_{i}=1-\exp \left(\frac{\sigma_{i}^{2} \times \log (0.5)}{\sigma_{0.5}^{2}}\right)
$$

Figure 1 shows the weighting term derived for two rectified key images with $\sigma_{0.5}=10$, demonstrating that the weight is reduced in regions of low image texture where stereo correlation will fail. 


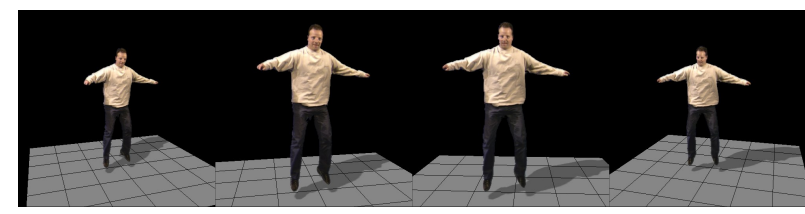

(a) Multiple novel views at a single time instant

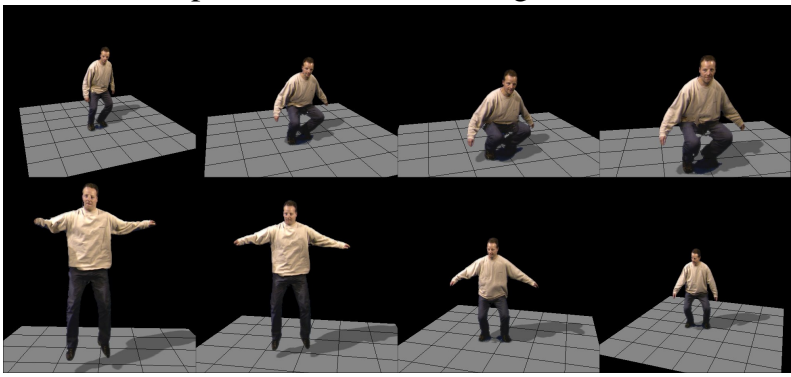

(b) Panning virtual camera for a jump sequence

Figure 2. Model-free multiple view optimisation from 8-camera views

\section{Model-free Optimisation}

In this section we demonstrate the application of the multiple view optimisation framework to image sequences without an a priori model of scene structure.

\subsection{Methodology}

An initial surface approximation is constructed from an implicit surface triangulation of the discrete visual-hull. This initial surface approximates all scene features upto the resolution of the visual hull (approx. 1 pixel). Previous silhouette based algorithms for rendering novel views of a scene reproject the images to the desired view based on the visual-hull geometry to synthesise a novel view [20, 10]. Direct reprojection based on the visual-hull results in visual artefacts due to errors in the surface approximation which cause misalignment between the images. In this work we use the multiple view optimisation framework to reconstruct a surface which gives sub-pixel alignment of images in regions of non-uniform appearance. Stereo correspondence precisely aligns those regions which have visible changes in surface appearance where misalignment would cause visual artefacts. Misalignment of overlapping images in regions of uniform appearance does not cause visible errors in the reprojected model except on the silhouette outline.

To reconstruct an approximate surface model in regions of uniform appearance we introduce a regularisation constraint into Equation 1 to enforce a smooth surface as in previous deformable surface approaches. The standard approach to shape regularisation is to treat a deformable model
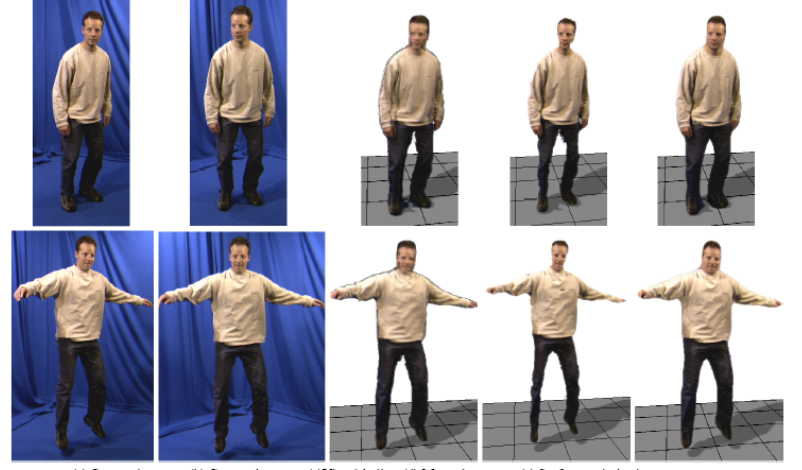

Figure 3. Comparison of techniques for view synthesis of an intermediate view mid-way between two cameras: (left) source images; (middle) Visual-hull [10, 20]; ( ${ }^{n d}$ right) Multiview stereo [12]; (right) Model-free optimisation (this paper)

as a thin-plate material under tension [19]. In this work we use a membrane tension for the regularisation term $E_{R}$ across a parameterised surface surface $\underline{x}(u, v)$ :

$$
E_{R}=\iint\left(\left\|\underline{x}_{u}\right\|^{2}+\left\|\underline{x}_{v}\right\|^{2}\right) d u d v
$$

The variational derivative is given by the Laplacian $\triangle(\underline{x})$. The Laplacian at a vertex of a regular mesh is given by the umbrella-operator in Equation 11 where the index $v$ spans the 1-neighbourhood $\underline{x}_{i, v}$ of a vertex $\underline{x}_{i}[18]$.

$$
\frac{d E_{R}}{d \underline{x}_{i}}=-\frac{1}{n_{i}^{v}} \sum_{v=0}^{n_{i}^{v}-1}\left(\underline{x}_{i, v}-\underline{x}_{i}\right)
$$

The membrane regularisation term ensures a smooth minimum area surface in regions of uniform appearance where no stereo or silhouette constraints are available.

\subsection{Results}

Model-free multiple view optimisation has been applied for novel synthesis in a blue-screen studio environment. Eight cameras were used for the results presented with seven cameras in an arc of $150^{\circ}$ and one camera overhead to constrain the visual-hull. The capture volume is approximately $3 \times 3 \times 2 m^{3}$ with approximately $1 m$ baseline between the arc of cameras for a viewing distance of $2-5 \mathrm{~m}$.

Figure 2 illustrates the reconstruction with multiple view optimisation of a jump. Multiple novel views are synthesised at a single time instant and a panning virtual camera taken at multiple instants throughout the sequence. Note 

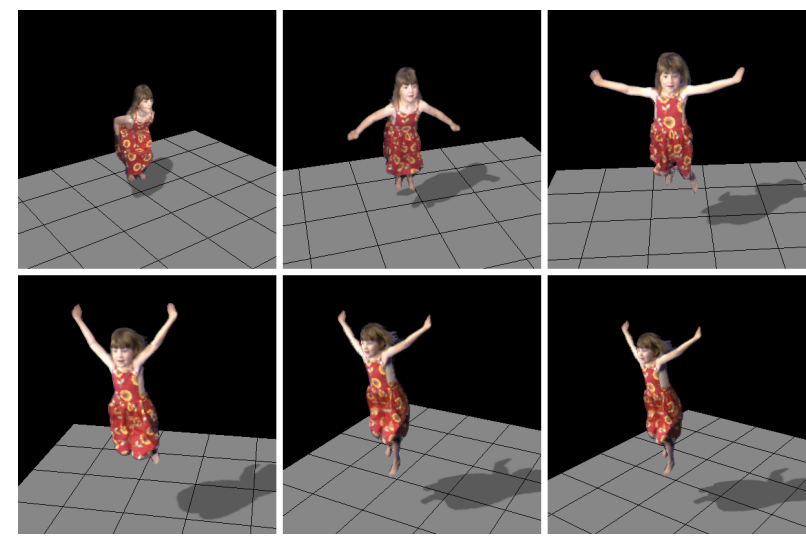

(a) Virtual view synthesis with model-free multiple view optimisation
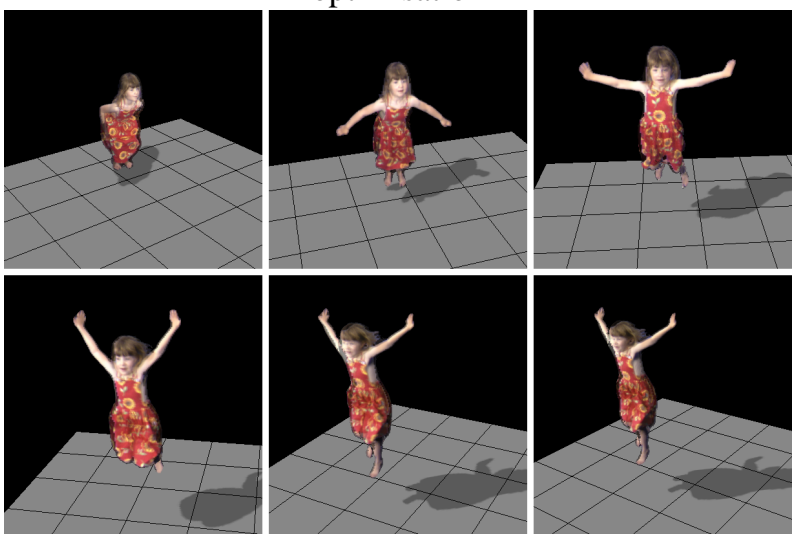

(b) Virtual view synthesis with visual-hull only

Figure 4. Virtual views with and without multiple view optimisation for six frames from an 8-camera video sequence of a jump

the reconstruction of the creases on the clothing throughout the sequence. A comparison of methods for novel viewsynthesis is presented in Figure 3 View-synthesis using the visual-hull [10, 20] results in loss of fine detail due to incorrect image correspondence resulting in blur. Stereo reconstruction [12] results in preservation of fine detail but fails to reconstruct regions near the silhouette. The multiple view optimisation presented in this paper overcomes the limitations of visual-hull or stereo to reconstruct a virtual view which reconstructs both fine detail and the silhouette outline.

Figures 4 and 5 present two more complex sequences of a girl with long hair and a dress jumping and performing a forward role. Such sequences would be difficult to either track or reconstruct with a model-based approach due to occlusion of the limbs. The model-free approach reconstructs the non-rigid dynamics of both the hair and dress. Comparison with the direct rendering using the visual-hull for

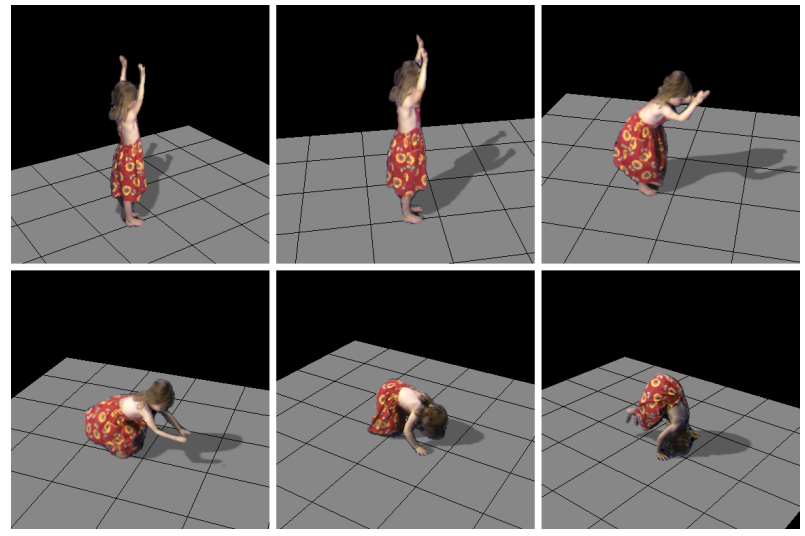

(a) Virtual view synthesis with model-free multiple view optimisation
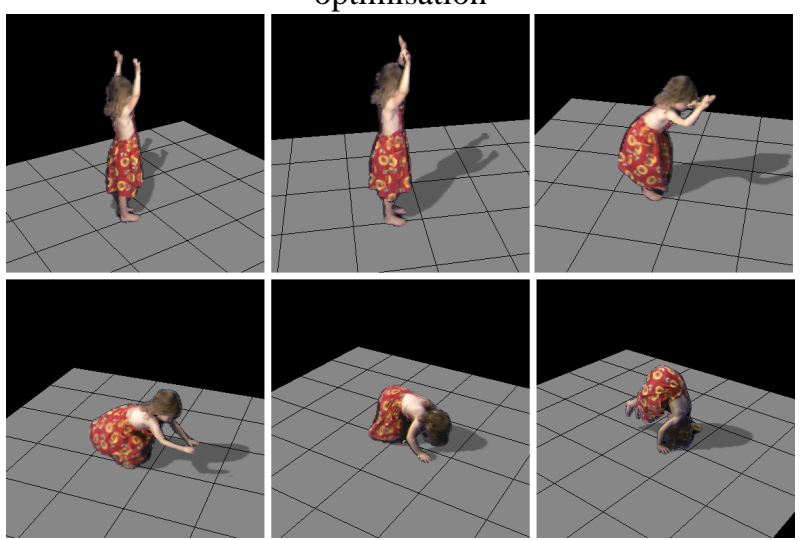

(b) Virtual view synthesis with visual-hull only

Figure 5. Virtual views with and without multiple view optimisation for six frames from an 8-camera video sequence of a forward role

the same sequences demonstrates the improvement in visual quality. The visual-hull exhibits artefacts on the face and visible misalignment of the pattern on the dress. The multiple view optimisation achieves novel view synthesis with a visual quality comparable to the captured video images. The stereo constraint ensures that regions of non-uniform surface appearance results in correct alignment of the pattern avoiding visual artefacts and blurring.

\section{Model-based Optimisation}

Model-based optimisation introduces a priori knowledge of the scene structure in the form of a generic model. This assumes that the observed scene approximates the generic model geometry. The advantage of model-based optimisation is the reconstruction of a consistent structure at each time instant suitable for animation. 


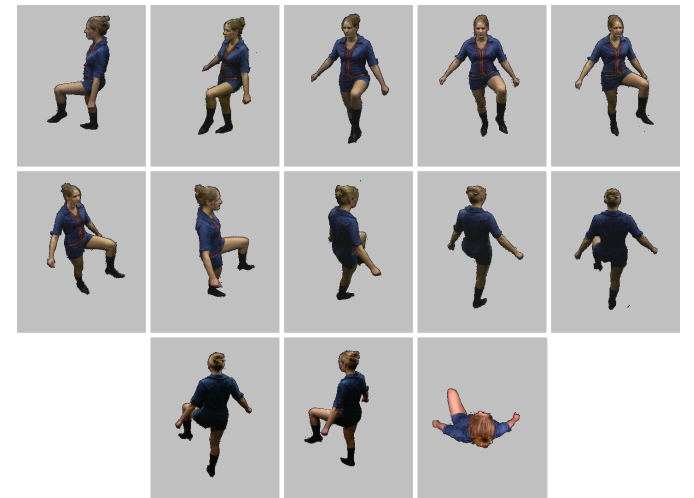

Figure 6. Thirteen camera views captured in the multiple camera studio.

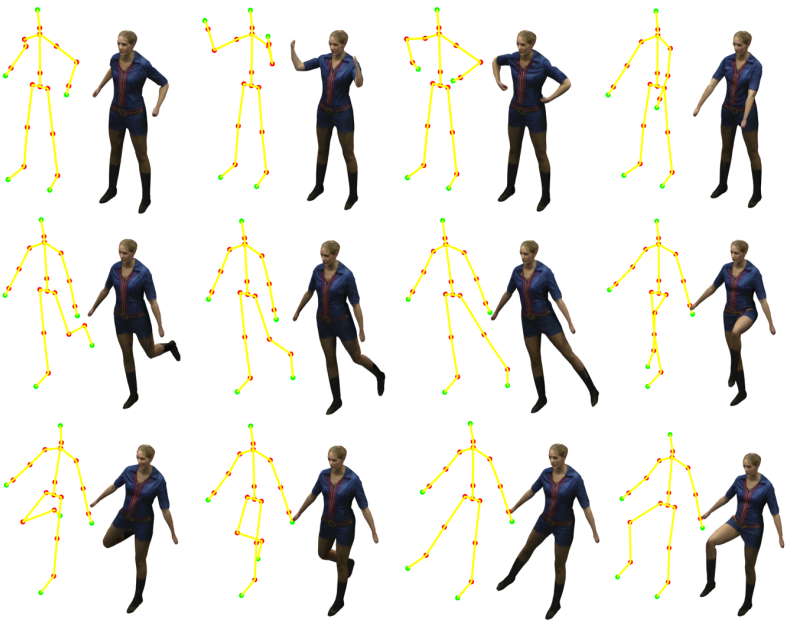

Figure 7. Models reconstructed for a range of different poses.

\subsection{Methodology}

In this work we assume a generic humanoid model whose pose is approximately aligned with the pose of the observed person via tracking or manual intervention. The generic model used in this work consists of a single seamless mesh defining the surface shape of the body, connected to a skeleton structure for animation. The mesh contains 8000 polygons and the skeleton 17 articulated joints, providing the gross shape and pose of the human body. A vertex weighting scheme is used for animation of the mesh surface based on the skeletal pose.

The internal energy of the model is designed to preserve the prior shape information of the generic model in order to regularise shape fitting in the presence of noisy or irregular data. Preservation of the local mesh parameterisation with respect to the underlying skeletal structure

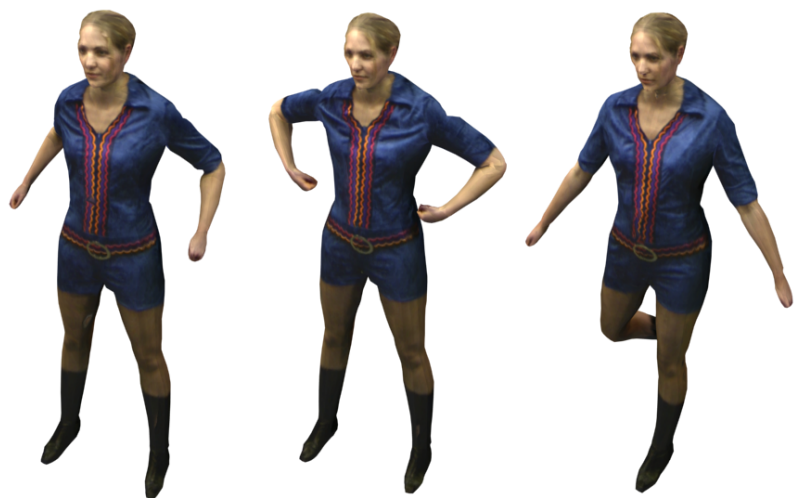

Figure 8. Enlarged images showing models reconstructed for different poses.

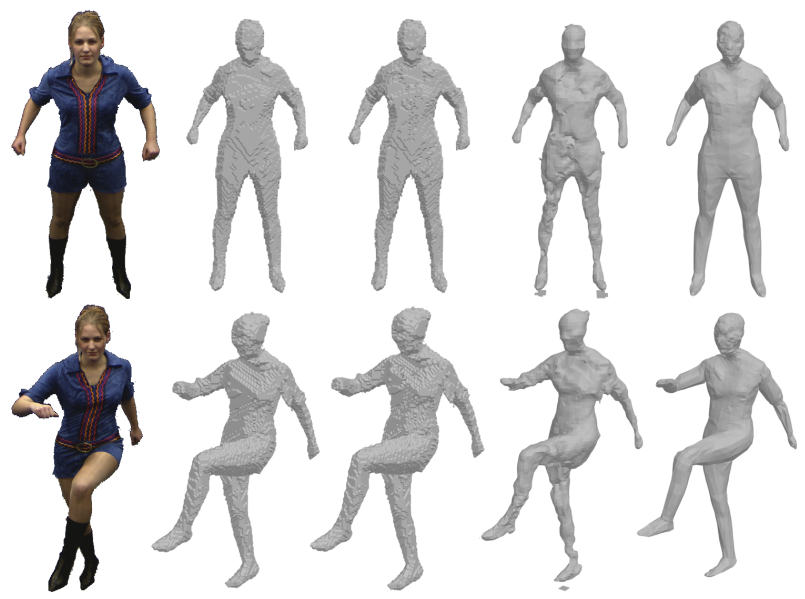

Figure 9. Comparison of shape reconstruction [15]: (left) images; ( $2^{\text {nd }}$ left) visual-hull; (middle) voxel-colouring; ( $2^{\text {nd }}$ right) merged stereo; (right) model-based, this paper

is essential for animation of the resulting model. A local shape constraint which preserves the parameterisation of arbitrary triangulated meshes was introduced in previous work [15]. This constraint is used to define the regularisation energy for the model surface as the squared error between the estimated surfaces and the original local shape. Equation 12, defines the model-based shape regularisation where $\left(\alpha_{v, j}, \beta_{v, j}, h_{v, j}\right)$ are the barycentric coordinates $(\alpha, \beta)$ and height offset $h$ in the $j^{t h}$ triangle-based frame for a vertex with valence $N_{v}$ on the generic model.

$$
E_{R}=\sum_{v} \frac{1}{N_{v}} \sum_{j}\left\|\underline{x}\left(\alpha_{v, j}, \beta_{v, j}, h_{v, j}\right)-\underline{x}_{v}\right\|^{2}
$$




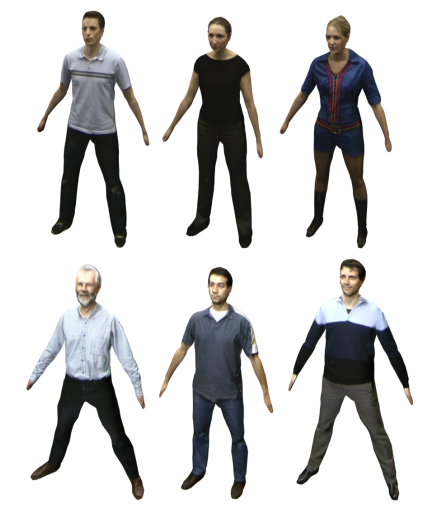

Figure 10. Six different models generated from 13 camera views in a studio.

$$
\frac{d E_{R}}{d \underline{x}_{v}}=-\frac{1}{N_{v}} \sum_{j}\left(\underline{x}\left(\alpha_{v, j}, \beta_{v, j}, h_{v, j}\right)-\underline{x}_{v}\right)
$$

The resulting reconstructed surface preserves the shape of the generic model in regions of uniform appearance and minimises the stereo reprojection error in non-uniform regions according to equation 1 . The model-based shape regularisation defined by equation 12 has the advantage of preserving the model shape in the presence of visual ambiguity rather than reconstructing a smooth surface approximation as is the case in the model-free approach.

\subsection{Results}

For the model-based reconstruction a set of 13 cameras in a chroma-key studio were used distributed uniformly around the subject as shown in Figure 6. The capture volume is approximately $2 \mathrm{~m}^{3}$ with 12 cameras uniformly spaced in a circle of radius $5 \mathrm{~m}$ around the volume and one overhead. This gives a stereo baseline of approximately $2.5 \mathrm{~m}$ between adjacent views.

Figure 7 shows the reconstructed models for twelve different poses of a person. Three close-ups of the reconstructed models are shown in Figure 8 The reconstructed models correctly align the texture between multiple views despite the relatively wide stereo baseline between views. Fine detail such as the fingers are not correctly reconstructed due to the image resolution. Visible artefacts occur at the shoulders due to the limitations of the vertex weighting scheme in animating the mesh surface. Figure 9 presents a comparison of the model-based multiple view reconstruction with previous approaches. This comparison demonstrates that the approach presented in this paper to combine stereo and silhouette correctly reconstructs both the detailed surface geometry and body shape.

Further model-based reconstruction results are presented in Figures 10. Results demonstrate the correct reconstruc-
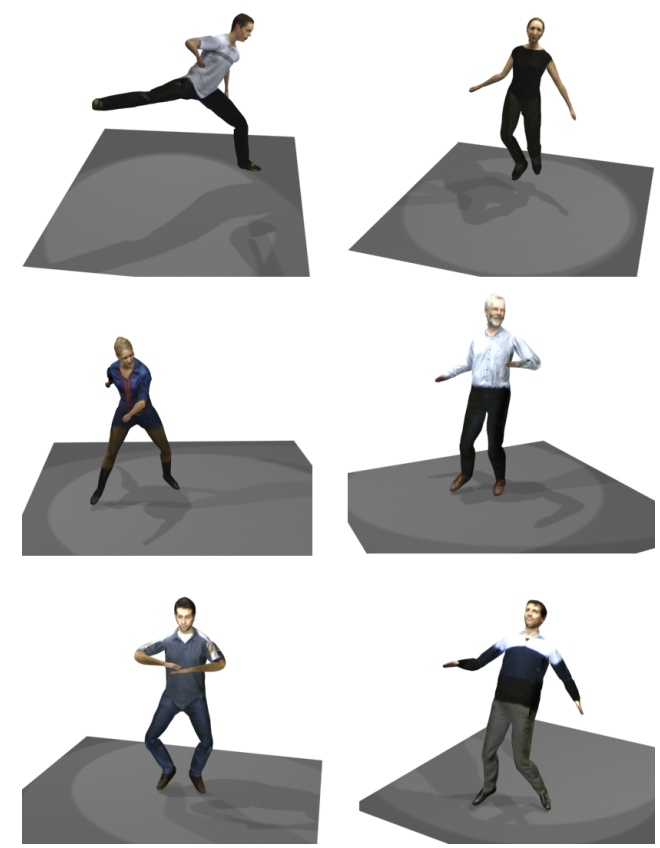

Figure 11. Animation of the reconstructed 3D models

tion of both shape and appearance for people wearing a variety of clothing. The model texture is correctly aligned between multiple wide-baseline stereo views. However, subjects are restricted to wearing clothing such that all limbs are visible to conform to the constraints of the generic humanoid model. The reconstructed models have a common articulated structure which can be animated, as illustrated in Figure 11 .

\section{Discussion \& Conclusions}

In this paper we have presented a framework for multiple view optimisation which has been applied to both model-free and model-based reconstruction. The optimisation framework combines silhouette and stereo constraints with a shape regularisation term based on prior assumptions of scene geometry. The stereo constraint includes a search for correspondence between multiple views to avoid convergence to the nearest local minima associated with previous gradient based multiple view stereo algorithms [4, 3].

Model-free reconstruction demonstrates novel view synthesis of complex dynamic scenes. In this work the modelfree approach is initialised at each time instant using the visual-hull surface. This surface is then optimised to satisfy the multiple view stereo and silhouette constraints together with a surface smoothness regularisation term to approximate the surface in regions of visual ambiguity. This over- 
comes limitations of previous visual-hull [10, 20] and multiview stereo [8] techniques by combining both constraints. The resulting novel views reproduce the complex dynamics of clothing and hair with a visual quality comparable to the captured video sequences. Model-free reconstruction has the advantage of requiring only weak assumptions on the observed scene structure. In this work, local surface continuity internal to the visual hull. This approach can therefore reconstruct multiple isolated objects and complex non-rigid dynamics. The principal limitation of model-free reconstruction is the lack of a common temporal structure for the reconstructed scene. This limits the modification and reuse of the captured scene to replaying the observed dynamics.

Model-based reconstruction introduces a priori knowledge of the scene structure to provide strong constraints on the observed scene. In this work a generic humanoid model comprising a seamless surface with an underlying articulated skeleton is used. Multiple view optimisation is then applied to fit the generic model to the observed stereo and silhouette. A local shape constraint is used to preserve the local mesh shape and parameterisation of the model during fitting. Preservation of the mesh parameterisation is essential to reconstruct a model which can be re-animated. Application of the model-based approach to multiple view reconstruction demonstrates the reproduction of high-quality models of people with correct alignment of textures between multiple relatively wide-baseline stereo views. The advantage of the model-based approach is that a common structured model is obtained at each time instant which can be re-animated in a standard computer graphics pipeline and provides an efficient representation. However, the modelbased reconstruction has two principal limitations. Firstly the requirement for an initial estimate of the model pose. This can be obtained from tracking or manual intervention. Automatic tracking of complex human movement with selfocclusion remains an open-problem. The second problem is the constraints imposed by the assumed model structure on the persons clothing and hair. Scene geometry which does not conform to the model structure is not correctly reconstructed due to the shape constraints in the multiple view optimisation.

This paper presents a common framework for modelbased and model-free multiple view optimisation. Results highlight the potential advantages and limitations of each approach. Overcoming these limitations remains the subject of future work.

\section{Acknowledgements}

This work was supported by EPSRC GR/S27962 and $\mathrm{PhD}$ research of Dr. Jonathan Starck [14] EPSRC DTI Broadcast Link PROMETHEUS Project GR/M88075 in collaboration with BBC R\&D.

\section{References}

[1] B. Allen, B. Curless, and Z. Popovic. Articulated body deformation from range scan data. In Proc. ACM SIGGRAPH, pages 612-619, 2002.

[2] J. Carranza, C. Theobalt, M. Magnor, and H.-P. Seidel. Freeviewpoint video of human actors. In Proc. ACM SIGGRAPH, pages 565-577, 2003.

[3] O. Faugeras and R. Keriven. Variational principles, surface evolution, pde's, level set methods and the stereo problem. Technical Report 3021, INRIA, 1996.

[4] P. Fua and Y. Leclerc. Object-centered surface reconstruction: Combining multi-image stereo and shading. International Journal of Computer Vision, 16:35-56, 1995.

[5] A. Hilton, D. Beresford, T. Gentils, R. Smith, W. Sun, and J. Illingworth. Whole-body modelling of people from multiview images to populate virtual worlds. Visual Computer: International Journal of Computer Graphics, 16(7):411—436, 2000. pdf

[6] X. Ju and J. Siebert. Conformation from generic animatable models to $3 \mathrm{~d}$ scanned data. In International conference of $3 D$ Scanning, Paris.

[7] I. Kakadiaris and D. Metaxas. Three-dimensional human body model acquisition from multiple views. International Journal of Computer Vision, 30(3):191-218, 1998.

[8] T. Kanade. Virtualized reality: putting reality into virtual reality. In 2nd International Workshop on Object Representation for Computer Vision ECCV, 1996.

[9] T. Kanade and P. Rander. Virtualized reality: Constructing virtual worlds from real scenes. IEEE MultiMedia, 4(2):3447, 1997.

[10] W. Matusik, C. Buehler, R. Raskar, S. Gortler, and L. McMillan. Image-based visual hulls. Proceedings of ACM SIGGRAPH, pages 369-374, 2000.

[11] S. Moezzi, L.-C. Tai, and P. Gerard. Virtual view generation for 3d digital video. IEEE MultiMedia, 4(2):18-26, 1997.

[12] P. Narayanan, P. Rander, and T. Kanade. Constructing virtual worlds using dense stereo. IEEE International Conference on Computer Vision, pages 3-10, 1998.

[13] R. Plankers and P. Fua. Articulated soft objects for videobased body modeling. IEEE International Conference on Computer Vision, pages 394-401, 2001.

[14] J. Starck. Human Modelling from Multiple Views. PhD Thesis, University of Surrey, 2003. pdf

[15] J. Starck and A. Hilton. Model-based multiple view reconstruction of people. In IEEE International Conference on Computer Vision, pages 915-922, 2003. pdf

[16] J. Starck and A. Hilton. Towards a 3d virtual studio for human apperance capture. In IMA International Conference on Vision, Video and Graphics, Bath, 2003.

[17] C. Stretcha, T. Tuytelaars, and L. Gool. Dense matching of multiple wide-baseline views. In International Conf. on Computer Vision, pages 1194-1201, 2003.

[18] G. Taubin. A signal processing approach to fair surface design. In Proc. ACM SIGGRAPH, pages 351-358, 1995.

[19] D. Terzopoulos, A. Witkin, and K. Kass. Constraints on deformable models: Recovering 3d shape and nonrigid motion. Artificial Intelligence, 36(1):91-123, 1988.

[20] S. Vedula, P. Rander, H. Saito, and T. Kanade. Modeling, combining, and rendering dynamic real-world events from image sequences. Proceedings of Virtual Systems and Multimedia, pages 323-344, 1998. 